\title{
From Evidence to Practice: Importance of Developing Guidelines for Clinical Practice in Migraine Prophylaxis with Acupuncture
}

\author{
Yemeng Chen* \\ New York College of Traditional Chinese Medicine, USA
}

*Corresponding author: Yemeng Chen, New York College of Traditional Chinese Medicine, USA.

\begin{abstract}
In acupuncture practice, emphasis was focused on episodic migraine treatment, but the evidence-based practice revealed that acupuncture in migraine prophylaxis is superior to conventional medicine which is significant to clinical practice. Body constitution, syndrome differentiation, acupoints selection, needle techniques, stimulation modality, and treatment plan are the factors to be considered for developing guidelines of clinical practice for acupuncture in migraine prophylaxis.
\end{abstract}

Key words: Acupuncture; Guidelines of Clinical Practice; Migraine; Prophylaxis

\section{Introduction}

As one of the primary headache disorders, migraines are a common neurological condition which has caused tremendous burdens to patients and society. It was estimated that 1-year prevalence of incident migraine was $2.2 \%$ (1.5\% in men and $3.1 \%$ in women) and declined with age and approximately $21 \%$ of people with migraine had reported a diagnosis by a health care provider, and close to $12 \%$ were found to have severe disability related to migraines [1]. Acupuncture, an important modality of nonpharmacological pain management, has demonstrated its efficacy and safety in the treatment of migraines without aura. Acupuncture has been an effective treatment for episodic migraines, with recent evidence indicating that acupuncture is, in fact, superior to conventional medicine in migraine prophylaxis [2-8]. The evidence is acquired from high quality clinical trial design in randomized double-blind trials, but the majority of acupuncture treatment plan is aimed to a research purpose which is standardized with less points and no differentiation for individual patients $[9,10]$. It is quite different compared to the real world of clinical practice so it is important to develop guidelines for clinical practice in migraine prophylaxis which was emphasized in the previous textbooks or practical protocols.

\section{Discussion}

In 2014, China Association of Acupuncture \& Moxibustion revised the "Evidence-Based Guidelines of Clinical Practice with Acupuncture \& Moxibustion: Migraine" [11] which emphasized the recommendation of episodic migraine treatments such as bleeding technique, electro-acupuncture and even a pyro-acupuncture. For the clinical reliving period of migraine, the guidelines strongly recommended a comprehensive acupuncture-moxibustion treatment, which includes acupoints selection based on channel syndromes, mainly Shaoyang Channel, and Zang-fu organs syndromes differentiation which assisted with modalities of scalp 
acupuncture, electro-acupuncture, warming-needle moxibustion and bleeding technique, etc. It is important to develop clinical practice guidelines which aim to migraine prophylaxis since it is a significant outcome of evidence-based practice. The following is the considerations.

\section{Body Constitution}

In traditional Chinese medicine (TCM) practice, individual body constitution is an important factor to identify during comprehensive body assessment before treatment. For chronic migraine patient, certain type of body constitution is the main reason which causes repeated episodes. According to current survey and statistical analysis, migraine patients are more likely presenting Qi Stagnation Constitution, Blood Stasis Constitution, and Yin deficiency Constitution $[12,13]$. So, the migraine prophylaxis should consider this constitutional factor which follows the "treating the root" principle in traditional Chinese medicine.

\section{Syndrome Differentiation}

It refers to the current status of the patient who suffered from frequent migraine episodes by overall analysis at approaches of TCM way of assessment such as: general body discomfort, symptoms, signs of tongue features, and pulse phenomenon, etc. It is a way to explore the pathogenesis and to determine syndrome differentiation either in a catalog of Zang-fu Organ syndromes or Channel syndromes, the later one also depends on the outcome of palpation, local area or in a distance. Mostly those patients are identified as syndrome of the Liver-related system and/or Shaoyang Channel. Blood stasis and phlegm accumulation are main pathogenesis of migraine.

\section{Acupoints Selection}

Since the body constitution and syndrome differentiation are determined, it is not difficult for an acupuncturist to select appropriate points. As an experienced acupuncturist, palpation on the channels and certain acupoints (spots) to explore tender points (spots) is a key of efficacy. During each visit, those tender points (spots) could be various on the location and severity of tenderness. Some empirical points are also very effective [14]. A PET-CT study on the specificity of acupoints through acupuncture treatment in migraine patients demonstrated that higher brain metabolism showed after inserting needles on acupoints like Waiguan (SJ 6), Yanglingquan (GB 34) and Fengchi (GB 20) [15]. Such scientific study report is also kind a clue for acupoints selection.

\section{Needle Techniques}

Applying specific needle techniques are ways to enhance therapeutic effect according to patient's syndrome differentiation. The practitioners should perform those needle techniques carefully and gently to make patient not uncomfortable during the treatment session.

\section{Stimulation Modalities}

Besides traditional body acupuncture methods, other acupuncture-related modalities are also used for migraine prophylaxis which include ear acupuncture, scalp acupuncture, dry needling techniques, and electro-acupuncture [16-21]. So, we can choose appropriate methods especially when one method failed during practice.

\section{Treatment Plan}

Based on some clinical trials data, prophylaxis of episodic migraine needs repeat treatment for about 20 sessions, in a mode of every other day for 10 sessions with 9-day break or in a mode of two acupuncture sessions per week continuous for 3 months [4,22]. For women who experienced episodic associated with menstrual cycle, frequency of visits should be adjusted accordingly. Affordability and insurance reimbursement allowance are also the considerations to adjust the plan for acupuncture treatment sessions.

\section{Conclusion}

Although evidence of acupuncture in migraine prophylaxis is confirmed, guidelines of clinical practice are necessary to develop aim to therapeutic outcome.

\section{Acknowledgement}

None.

\section{Conflict of Interest}

Author declares no conflict interest.

\section{References}

1. Lipton RB, Reed ML, Munjal S (2019) One-year incidence of migraine in the US population: results from the Migraine in America Symptoms and Treatment (MAST) study. Headache 59(S1): 9-10.

2. Linde K, Allais G, Brinkhaus B, Fei Y, Mehring M, et al. (2016) Acupuncture for the prevention of episodic migraine. Cochrane Database of Syst Rev 2016(6): CD001218.

3. Zhao L, Chen J, Li Y, Sun X, Chang X, et al. (2017) The Long-term Effect of Acupuncture for Migraine Prophylaxis: A Randomized Clinical Trial. JAMA Intern Med 177(4): 508-515.

4. Xu S, Yu L, Luo X, Wang M, Chen G, et al. (2020) Manual acupuncture versus sham acupuncture and usual care for prophylaxis of episodic migraine without aura: multicentre, randomized clinical trial. BMJ 368: m697.

5. Fan SQ, Jin S, Tang TC, Chen M, Zheng H (2020) Efficacy of acupuncture for migraine prophylaxis: a trial sequential meta-analysis. J Neurol 24.

6. Ni X, Dong L, Tian T, Liu L, Li X, et al. (2020) Acupuncture versus Various Control Treatments in the Treatment of Migraine: A Review of Randomized Controlled Trials from the Past 10 Years. J Pain Res 13: 2033-2064.

7. Li YX, Xiao XL, Zhong DL, Luo LJ, Yang H, et al. (2020) Effectiveness and Safety of Acupuncture for Migraine: An Overview of Systemic Reviews. Pain Research and Management 2020: 3825617.

8. Zhao L, Zhang FW, Li Y, Wu X, Zheng H, et al. (2011) Adverse events associated with acupuncture: three multicenter randomized controlled trials of 1968 cases in China. Trials 12:87.

9. Ying L, Zhang H, Witt C, Roll S, Yu SG, et al. (2012) Acupuncture for migraine prophylaxis: a randomized control trial. CMAJ 184(4): 401-410. 
10. Liu L, Zhao LP, Zhang CS, Zeng L, Wang K, et al. (2017) Acupuncture as prophylaxis for chronic migraine: a protocol for single-blinded, double-dummy randomized controlled trial. BMJ OPEN 8(5): e020653.

11. China Association of Acupuncture \& Moxibustion (2014) Evidence-Based Guidelines of Clinical Practice with Acupuncture \& Moxibustion: Migraine. Press of Traditional Chinese Medicine, Beijing, China.

12. Li Z, Ni W, Zhao Y (2015) A Clinical Study on the Relationship between TCM Constitution and TCM Syndromes in 250 Patients with Migraine. Journal of Liaoning University of Traditional Chinese Medicine 17(10) 13-14.

13. Xie W, Ni W, Ni J (2016) Based on Syndrome and Constitution Explore TCM Pathogenesis and Staging Treatment of Migraine. Liaoning J of Traditional Chinese Medicine 43(9): 1855-1857.

14. Chen Y (2004) Introduction to Prof. You-An Fang's Empirical Acupoints Applied to Treating Migraine. Am J of Tradit Chin Medicine 5(2): 18-20.

15. Yang J, Zeng F, Fang Y, Fang L, Qin W, et al. (2012) A PET-CT study on the specificity of acupoints through acupuncture treatment in migraine patients. BMC Complement Altern Med 12: 123.

16. Chen Y, Liu B, Gong W, Guoqiang L (2020) Auricular acupuncture for migraine: A protocol for systemic review and meta-analysis. Medicine 99(44): e23036.
17. Ceccherelli F, Lovato A, Piana E, Gagliardi G, Roveri A (2012) Somatic acupuncture versus ear acupuncture in migraine therapy: a randomized, controlled, blind study. Acupunct Electrother Res 37(4): 277-293.

18. Rezvani M, Yaraghi A, Mohseni M, Fathimoghadam F (2014) Efficacy of Yamamoto new scalp acupuncture versus Traditional Chinese acupuncture for migraine treatment. J Altern Complement Med 20(5): 371-374.

19. Pourhmadi M, Mohseni-Bandpel M, Keshtkar A, Koes BW, Fernández-deLas-Peñas C, et al (2019) Effectiveness of dry needling from improving pain and disability in adults with tension-type, cervicogenic, or migraine headaches: protocol for a systemic review. Chiropr Man Therap 27: 43.

20. Li X, Dai Q Shi Z, Chen H, Hu Y, et al. (2019) Clinical Efficacy and Safety of Electroacupuncture in Migraine Treatment: A Systemic Review and Network Meta-Analysis. Am J Chin Med 47(8): 1755-1780.

21. Jia CS, Ma XS, Shi J, Wang YM, Li YF, et al. (2009) Electroacupuncture at Qiuxu (GB 40) for treatment of migraine - a clinical multicentral random controlled study. J Tradit Chin Med 29(1): 43-49.

22. Zhang XZ, Zhang L, Guo J, Zeng L, Yang Y, et al. (2013) Acupuncture as prophylaxis for menstrual-related migraine: study protocol for a multicenter randomized controlled trial. Trial 14: 374. 